\title{
Factual Revelation of Temporal and Spatial Hierarchical Correlations by Structural Function Curvature Analysis Method
}

\author{
Grigory V. Vstovsky ${ }^{1}$ \\ 1 “Energoavtomatika” Ltd., Moscow, Russia \\ Correspondence: Grigory V. Vstovsky, "Energoavtomatika” Ltd., 3/2, Ostapovsky Pr., 109316, Moscow, Russia. \\ Tel: 7-495-981-0136. E-mail: vstovsky@yandex.ru
}

Received: March 1, 2012 Accepted: March 26, 2012 Online Published: May 24, 2012

doi:10.5539/esr.v1n2p30 URL: http://dx.doi.org/10.5539/esr.v1n2p30

\begin{abstract}
Basics of structural function curvature analysis method (SFCAM) are described shortly and three examples of SFCAM application are described: for revelation of earthquake predictors, surface relief analysis and surface relief evolution description. Discussion is carried out in terms of correlation times (CT) or correlation lengths (CL), respectively to the type of experimental data - time series or surface reliefs. CTs or CLs can be taken into account in two ways: by an order of their recognition, from the shortest to the greatest, or by separation over previously determined classes due to physical sense of investigated structure. On the whole, SFCAM represent a subtle enough tool for analysis of complex hierarchical systems.
\end{abstract}

Keywords: time series, surface relief, complexity, structural function, correlation time, correlation length

\section{Introduction}

This short review is to represent a new perspective tool for data processing in Earth's science: time series and surface reliefs. Time series are usually obtained when investigating the earthquakes, volcano activity and other natural phenomena producing vibrations that can be recorded in some digital form and then characterized quantitatively on the base of one or another mathematical apparatus. Surface reliefs are basic data for morphological analysis. They can be also scanned and recorded digitally to be visualized and mathematically processed and described. Most of modern quantitative description techniques for real data lean upon statistical analysis of measured values (the heights, sizes and other local or distributed parameters) giving their maximum, mean and minimum values, standard deviation, etc. - the parameters that can be easily calculated, but giving no information about "internal structure" of the investigated object represented by the data under analysis. Meanwhile, all the real systems have, as a rule, highly organized hierarchical structures with internal correlations in time and space. And evolution of such systems, first of all, shows itself in changes and alterations of these correlations. A lot of general information on these topics can be found elsewhere, for example, Timashev (2011), where the basics of a so called Flicker Noise Spectroscopy (FNS) are described, from which the ideas described in this paper had grown.

Structural function curvature analysis method (SFCAM) offers a new tool of quantitative description of complex natural systems in terms of hierarchy of correlation times or lengths (CT, CL), first proposed in (Vstovsky, 2006). Of course, these values can't be calculated exactly for real "living" systems and we have to apply statistical methods to evaluate CTs and CLs, but these are statistics of hierarchy parameters. All these features are described below. First, the basic mathematical procedure is described, as shortly and simply as possible, applied to single series processing. Then application to time series analysis is described for revelation of earthquake precursor (Bornyakov \& Vstovsky, 2010; Vstovsky \& Bornyakov, 2010). Finally, application of SFCAM to surface relief analysis is demonstrated (Vstovsky, 2006). Surface reliefs, being more complex objects as compared with time series, require the use of additional statistical methods, because relief is treated as the set of spatial series, and one has to process each its series separately and then average out parameters of the series over all the relief. Nevertheless, as is shown below, such a complicated approach can be used even to follow relief evolution (Vstovsky et al., 2006).

\section{SFCAM Basics}

Structural function (SF) represents an effective tool in theoretical and experimental investigations of chaotic systems and processes, for example, in turbulence investigations (Frisch, 1995). For the series $\left\{h\left(t_{k}\right)\right\} \operatorname{SF}$ of order $p$ is calculated by formula 


$$
\Phi^{p}(\Delta)=\frac{1}{M} \sum_{k=1}^{M}\left|h\left(t_{k}\right)-h\left(t_{k}+\Delta\right)\right|^{p},
$$

where $h(t)$ - signal value in the series of $N$ samples («points»), signal being given discretely in points (moments) $t_{k}=k \Delta t(\Delta t-$ discretization step) along coordinate $t, M=N-\Delta / \Delta t, \Delta-$ lag (argument) of SF equal to $\Delta t, 2 \Delta t, 3 \Delta t, \ldots$, $\left(N_{\Phi}\right) \Delta t, N_{\Phi}=(0.5-0.8) N, p-\mathrm{SF}$ order. $p=2$ is used as a rule and this index will be omitted for this case. Otherwise $p$ value is given explicitly. Figure 1 explains SFCAM. It shows examples of signal piece, its SF (in double logarithmic axes) and corresponding negative second derivative ("curvature") of smoothed double logarithmic SF. Extremuma positions of the latter give evaluations of CTs.
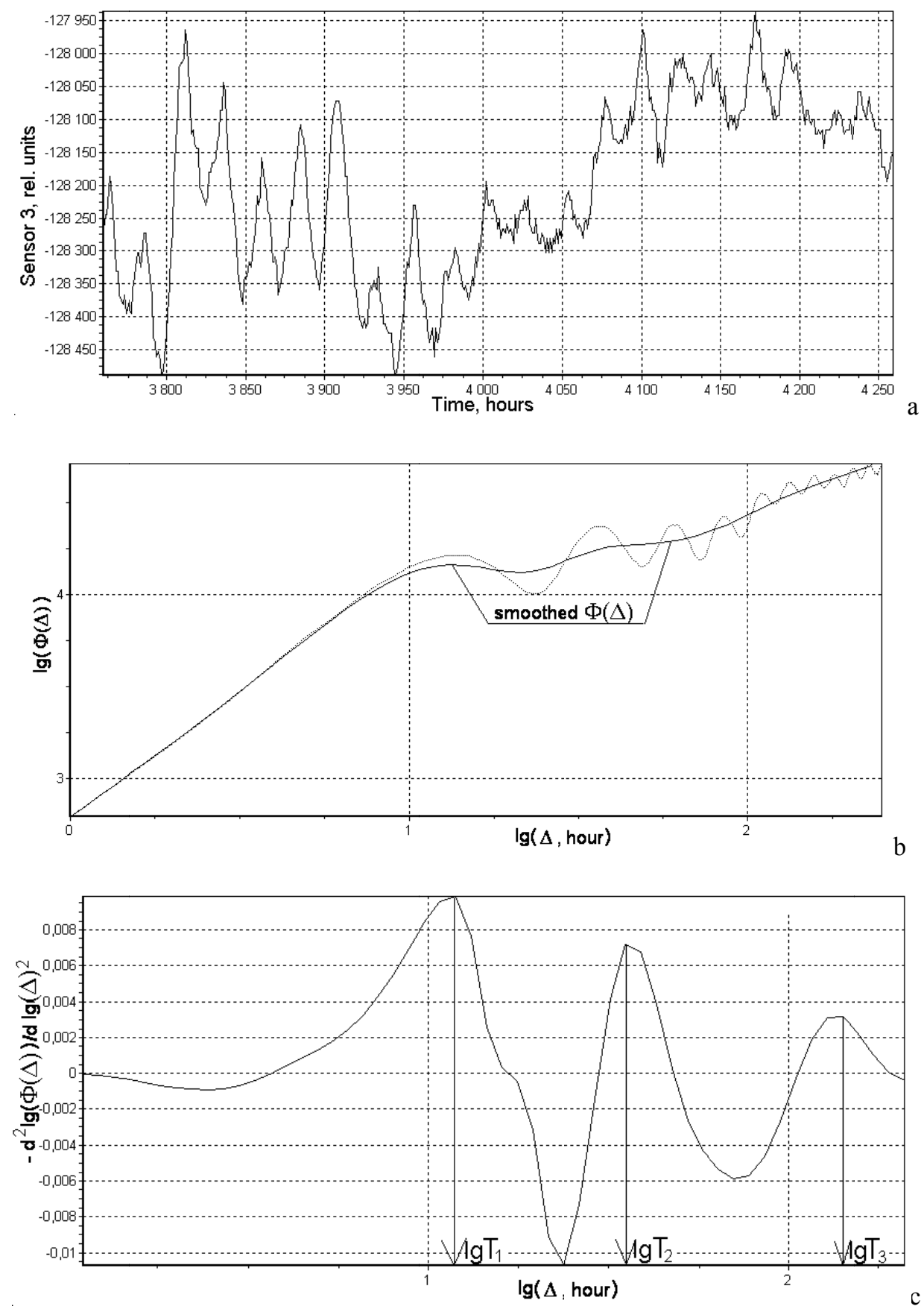

Figure 1. Piece of a signal (a), its double logarithmic SF and smoothed double logarithmic SF (b) and negative second derivative ("curvature") of smoothed double logarithmic SF (c) with indication of extremuma positions 
Physical sense of SF is as follows. SF growth for small lags $\Delta$, see Figure $1 \mathrm{~b}$, means that there are correlation links, interrelations, in the signal. Cessation of SF growth, saturation, for large lags means a failure (lost) of the interrelations. Conventional boundary of these scale regions (determined by one or another rule or algorithm) is called a correlation time (CT). In the simplest cases, when SF has only one "footstep", there is only one type of interrelation characterized by CT according to "footstep position" which can be determined by position of maximum of negative derivative of smoothed SF (under condition of positive first derivative) that was first proposed in (Vstovsky, 2006). That paper demonstrated effectiveness of such an approach in comparison with many parameter fitness (approximation) of SF. When SF has several "footsteps", corresponding to several different types of interrelations with different CTs, evaluation of SF's negative second derivatives extremuma positions enables to evaluate easily such CTs. So we can evaluate hierarchy of CTs. The case of SF with three "footsteps" is shown in Figure 1b, 1c. CTs are denoted by $T_{1}, T_{2}, T_{3}, \ldots$ starting with the smallest. This is a core of SFCAM.

Since real data are measured with finite accuracy, fluctuations, gaps and with other "defects", the calculated SFs are always imperfect, rough or deviating, one has to calculate CTs by a special multi-step algorithm including operations of reinterpolation of double logarithmic SF, smoothing it and then taking its second derivative. The used algorithms enable also to take into account the presence of data gaps (marked with a specified value) that is unique advance of the procedures used. These algorithms were coded in the program SHIFT (Scale Hierarchy Information Fertile Treatment) for time series analysis, and program sm2FNS for relief (atomic force microscopy) data processing. The results described below were obtained by these programs.

\section{Time Series Processing}

Processing of this data type is based on combination of SFCAM and sliding window method (SWM). The latter enables to present dynamical variable series as a sequence of values of one or another parameter calculated for each position of data window of given length. Window time coordinate is a position of its forward boundary. In such a way one can obtain the new time series of calculated parameters ascribing the parameters value to each window coordinate. For example, one can obtain the series of the parameters $T_{1}, T_{2}, T_{3}$, shown in Figure 1c, by sliding the window (piece), shown in Figure 1a, along a whole series of initial data.

This approach was used in refs (Bornyakov \& Vstovsky, 2010; Vstovsky \& Bornyakov, 2010; Vstovsky, 2008). The most demonstrative results obtained in such a way are shown in Figures 2, 3, 4. In (Vstovsky \& Bornyakov, 2010) the results were reported for revealing the precursors of an intensive South-Biakal earthquake $(M=6.3$, at 09:31 on 27 August 2008). The logic of CT hierarchy analysis application to earthquake prediction is as follows. It is implied (Vstovsky, 2008) that, as the processes take place on/in the distributed self-organized system, the changes in the state of such a system must influence the course of the local processes. For instance, during the accumulation of deformation in the Earth's crust under external and internal factors, and as a consequence, the rearrangement of the crust space structure, CTs of local deformation processes can change. The registration and adequate interpretation of such changes can be an effective tool for the monitoring of the states of both the separate regions and the lithosphere as a whole to reveal and forecast the transition and critical states in the lithosphere evolution process. Several interesting results were reported in (Bornyakov \& Vstovsky, 2010; Vstovsky \& Bornyakov, 2010) about South-Biakal earthquake prediction, but the most significant are shown in Figures 2, 3, 4. Earthquake moment is marked with dotted line. CTs obtained with SF of second order, $p=2$, are shown in Figure 3, and the same values for $p=4$ (first reported here) are shown in Figure 4. One can see that more or less stable correlations with CTs about $T_{1}=10, T_{2}=40, T_{3}=140$ hours break apart, CTs decrease in more than 3 times, at 1700 hours (71 days) before the earthquake. Increase in SF order makes the precursor and earthquake indicator sharper.

The results were obtained for hourly data, and calculation time expenses are of order 1 second per one point (or lesser). So, the described mathematical apparatus represents a real tool for (or to be a part of) on-line monitoring of seismic activity, or other Earth crust processes. 


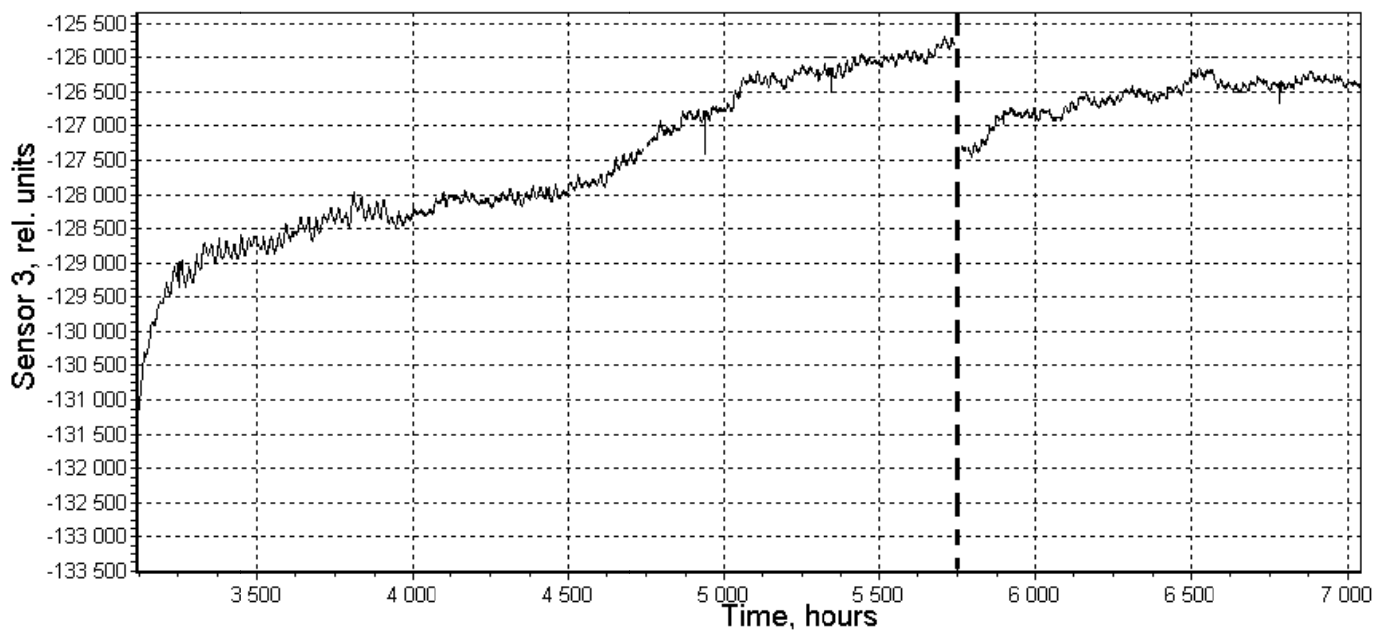

Figure 2. Recoded signal of vertical deformations in marblerock massive adit (Vstovsky \& Bornyakov, 2010)
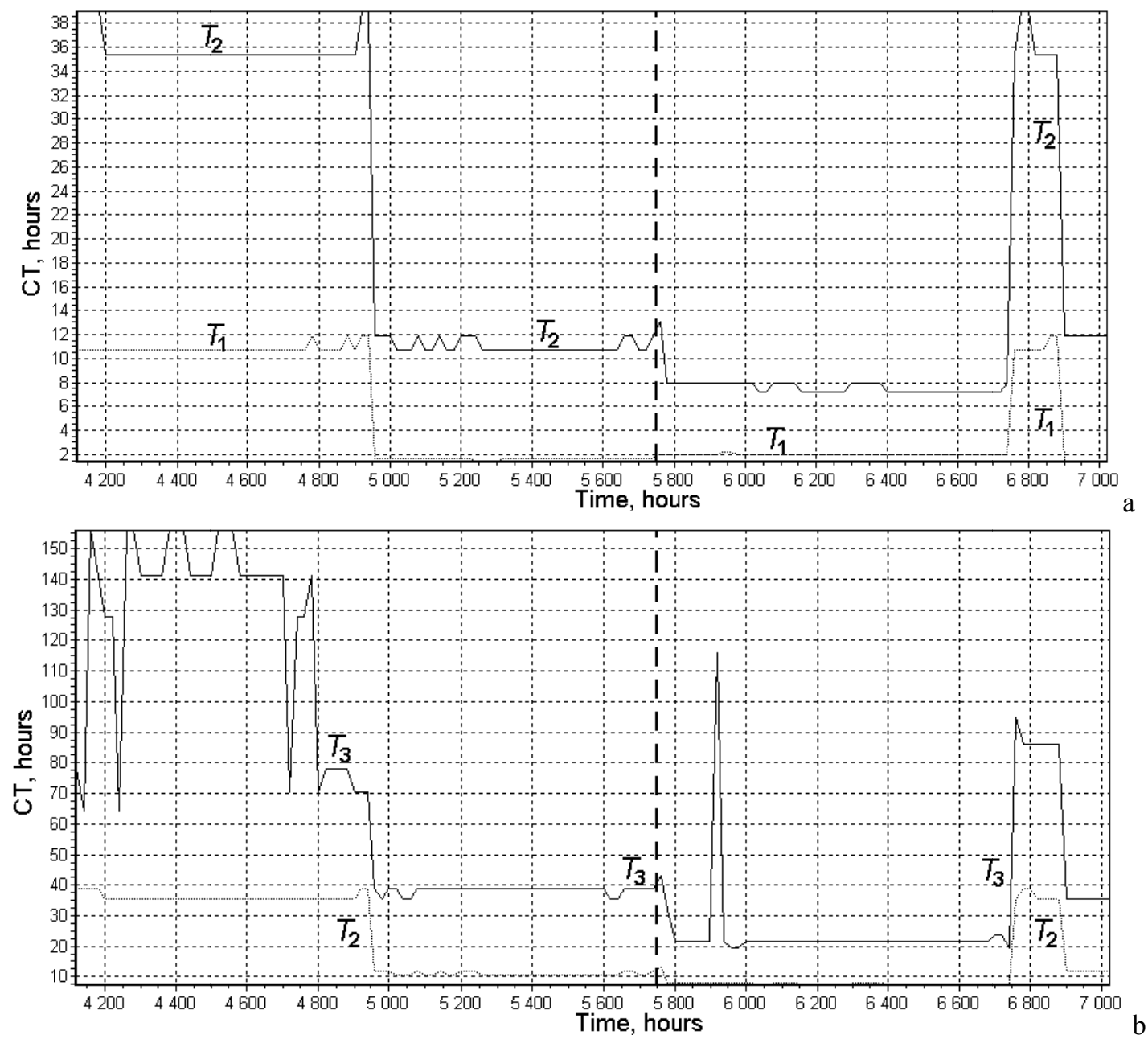

Figure 3. Results of calculation CTs $T_{1}, T_{2}, T_{3}$ for signal in Figure 2 for SF order $p=2$ and window size 1000 hours (Vstovsky \& Bornyakov, 2010). Earthquake is marked by dotted line 

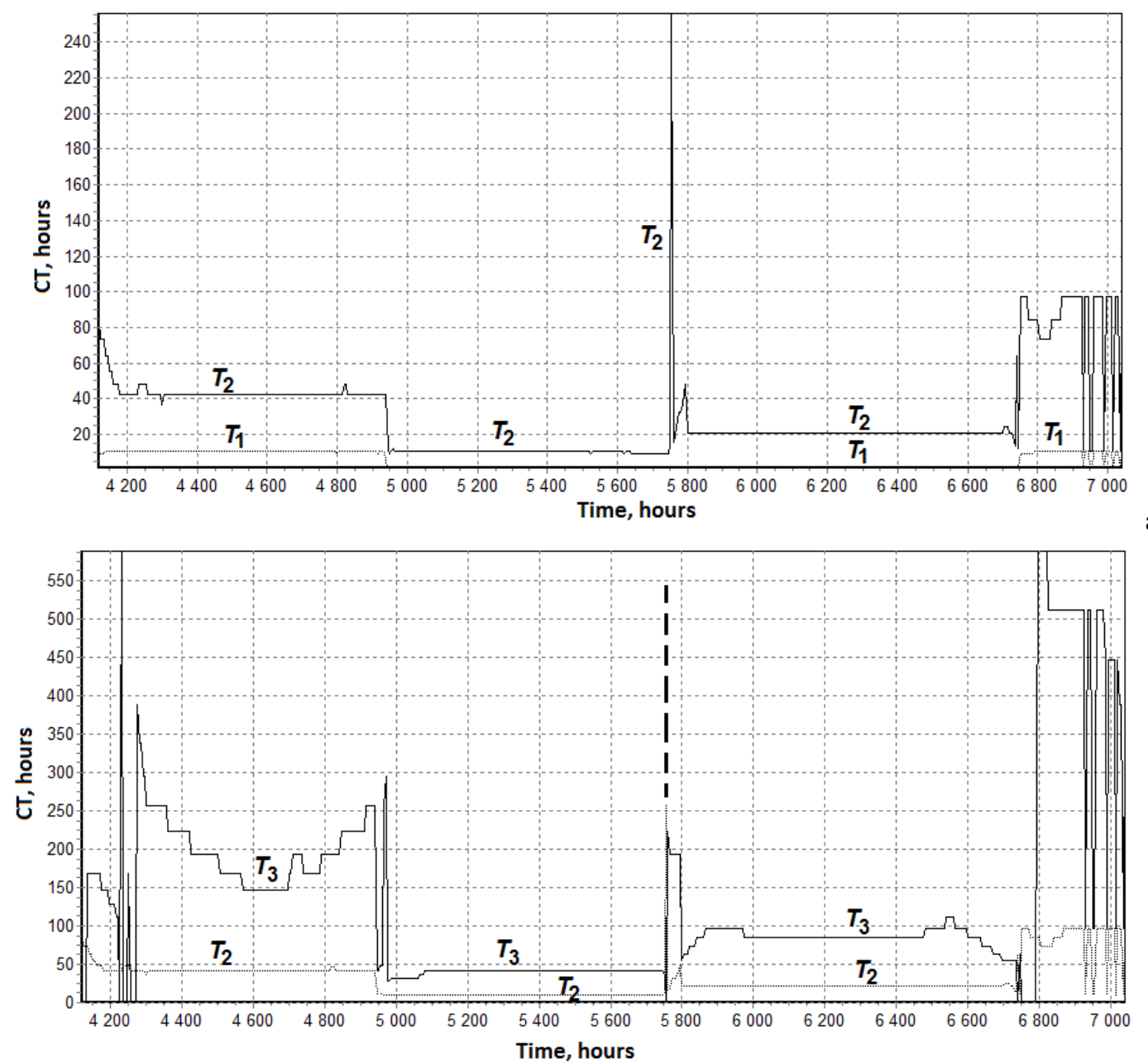

Figure 4. Results of calculation CTs $T_{1}, T_{2}, T_{3}$ for signal in Figure 2 for SF order $p=4$ and window size 1000 hours. Earthquake is marked by dotted line

\section{Surface Reliefs}

Application of SFCAM to relief processing is realized by processing the relief profiles in one or another direction, and following averaging the obtained profile parameters over relief. Two ways of averaging may be used, Figure 5, where "Curves" is for the SF and its negative derivative (other functions can be calculated also, for example, the Fourier power spectra). Below the results obtained by "Way 2" averaging are described.

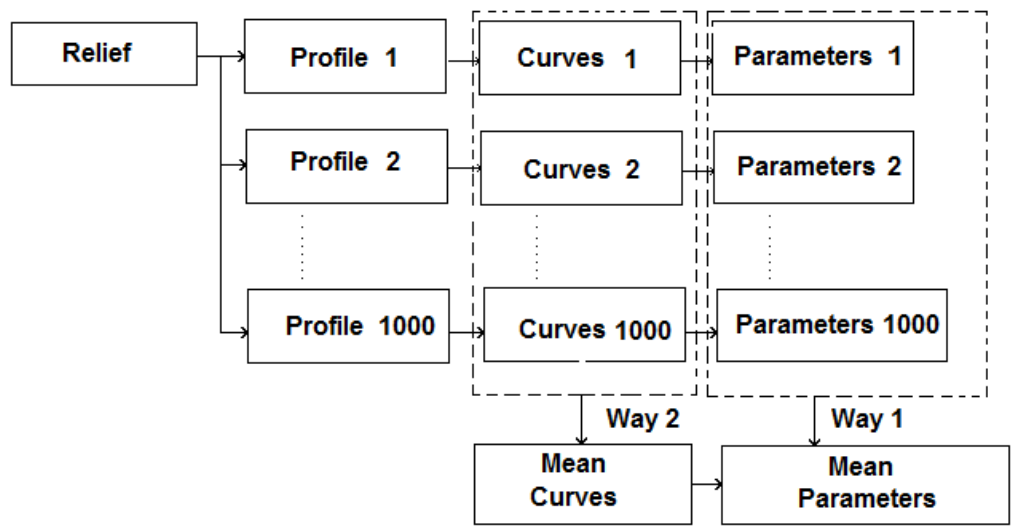

Figure 5. Two ways of obtaining average parameters for processing relief by SFCAM in "profile by profile" mode 
This is easily realized for reliefs of $1000 \times 1000$ points size or about, and the smaller relief the less reliable CLs values. First application of SFCAM to relief processing was described in ref. (Vstovsky, 2006), where this method was proved to be valid being tried out for model objects with known CL values also. The experiments, described in ref. (Letnikova et al., 2001), were to investigate an influence of hydrogen treatment (by digester at high temperature and pressure) on the dissolution surfaces of LiF monocrystals. A mirror technique was used when monocrystal was cleaved into two pieces with mirror surfaces, then one piece was treated, but the other was kept as a reference sample. Atomic force microscopy (AFM) data (contact mode, 50x50 mcm, 1000x1000 points) were obtained for the samples dissolved in the water at temperatures $25-50{ }^{\circ} \mathrm{C}$.

Typical relief view is shown in Figure 6. Typical views of the "curves" obtained for each relief are shown in Figure 7. These functions were averaged and parameters of average function were used to characterize the relief. More exactly, mean SFs were calculated for each relief and then CLs were obtained from their second derivatives. The arrows, indicating Cls $L_{1}$ and $L_{2}$ values, are given to explain the method. So, two CLs were revealed, Figure 8.

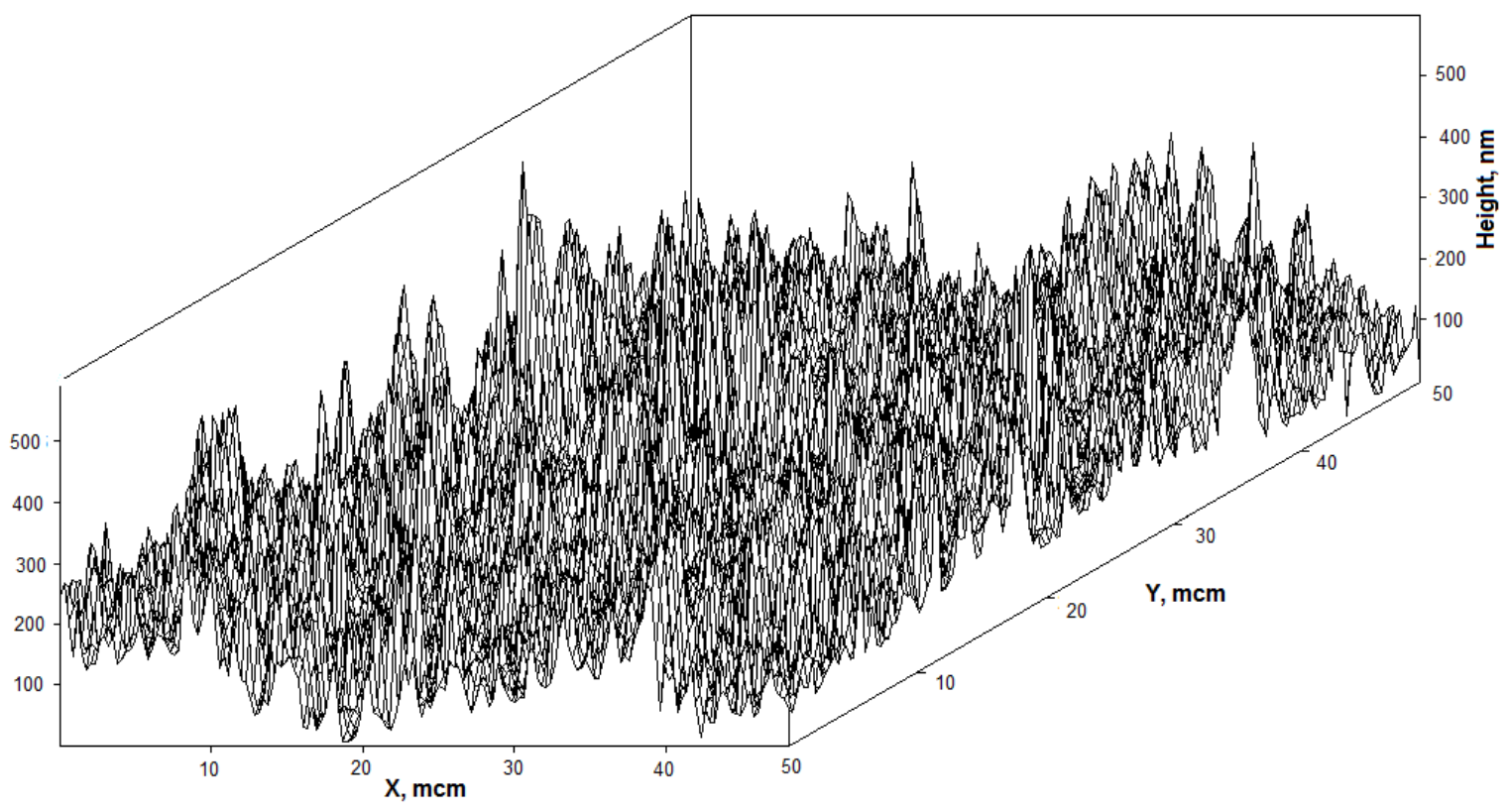

Figure 6. Typical view of LiF dissolution surface AFM relief (Each $6^{\text {th }}$ point is shown for both the directions $x, y$ )

The presence of two surface substructures is due to the way of monocrystal surfaces making - by cleavage generating the multiple crystal defects due to shear stresses. CL $L_{1}(300-800 \mathrm{~nm})$ corresponds to the dislocation lodgments. CL $L_{2}(10-25 \mathrm{mcm})$ corresponds to extrusion-intrusion heterogeneities appearing as the "steps" and "crests" on dissolution surfaces (Letnikova et al., 2001). Surface anisotropy is due to the latters. Increase in dissolution temperature diminished effect of hydrogen treatment on extrusion-intrusion heterogeneities.

Thus, SFCAM enables to follow the subtle features of the objects under investigations. Here, this is demonstrated for the structures at micrometer scale, but the very method and its algorithms can be generally applied for processing digital relief data, representing the real objects of any required size or nature, including abstract reliefs, for example, distributions of insect populations, microbiological characteristics, ozone concentration, magnetic field intensity, or whatever else. "Profile-by-profile" application of SFCAM can be applied also to digital 3D distribution data - to reveal anisotropy, for example. 

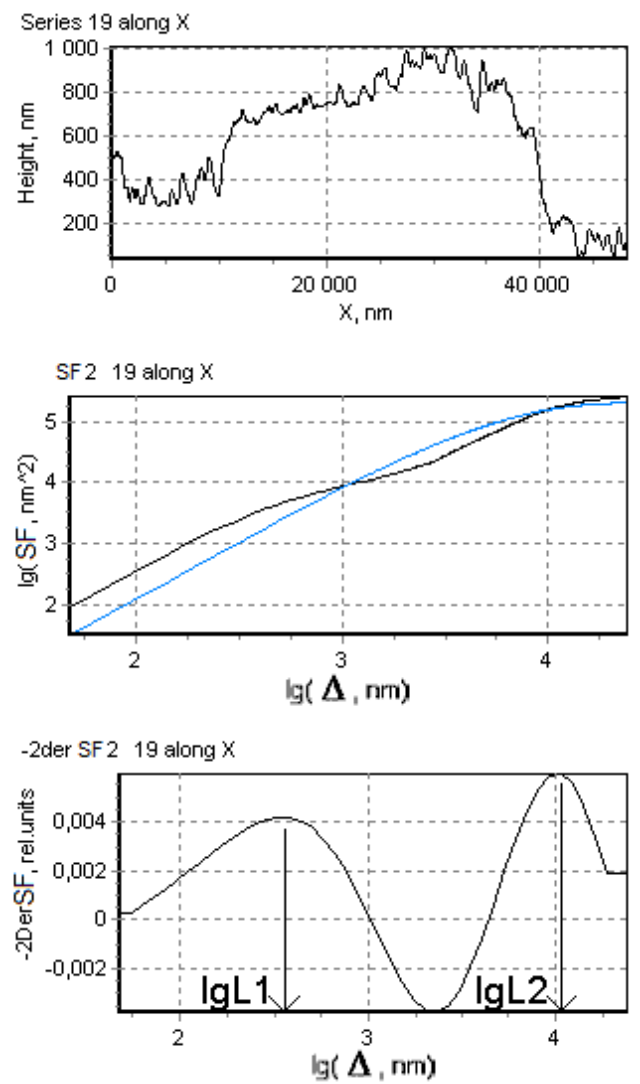

Figure 7. Typical views of SF and its negative second derivative which were calculated for each relief profile
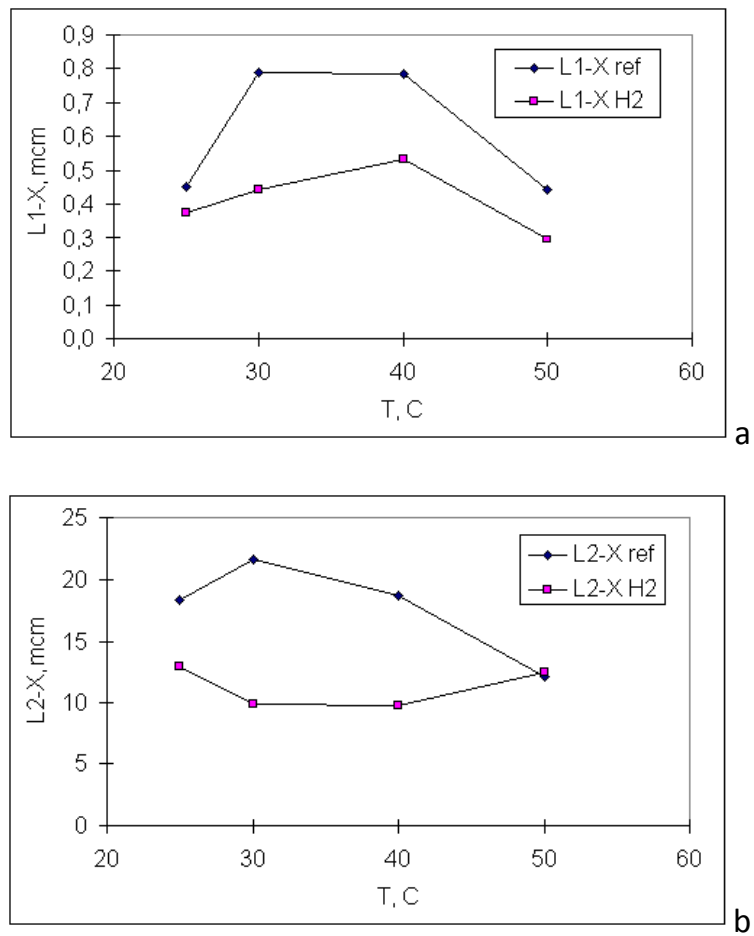

Figure 8. Dependence of CLs $L_{1}$ (a) and $L_{2}$ (b) on temperature for AFM data of ref. (Letnikova et al., 2001). "ref" (reference) is for initial crystal, " $\mathrm{X}$ " is for the direction of picked up profiles. Each value was obtained by SF averaged over 200 randomly picked up profiles and by averaging over 3-5 relief images 


\section{Surface Relief Evolution by Optical Microscopy Data}

Above, the resulted CLs values were taken into account due to the order of their recognition - from the smallest to the greatest. But in some applications one has to analyze CLs statistically by separation them into several classes due to physical sense of CLs appearance. The case of such an approach is described in this section.

In ref. (Vstovsky et al., 2006) the mechanochemical polymer coverings of led shot were subject to fast chemical corrosion tests and their surfaces were investigated by optical microscopy with following data processing on the base of SFCAM. The proposed approach can be used to control the polymer and other coverings (of various geometry) both in laboratory conditions and real exploitation conditions.

The experiments were as follows. The $3 \mathrm{~mm}$ led balls were covered with high density polyethylene (HDPE) by mechanochemical method in ball mill, the coverings having grained structure (with about $30 \mathrm{mcm}$ grains) due to the size of initial particles of HDPE dust introduced into the mill to form covering. Covering thickness was 50-100 $\mathrm{mcm}$.

Then covered balls were subject to accelerated chemical tests: the obtained covered balls were put into solution of potassium dichromate in $0.1 \mathrm{~N}$ hydrochloric acid, that will be referred to as chemical etching below. When covering solidness was destroyed, the insoluble lead chromate was produced, and potassium dichromate concentration decrease enabled to register corrosion process by subtle spectrophotometer method. In this case the effective destruction of coverings was observed after 7 days from tests start.

To conduct optical microscopy investigations, 3-5 balls were withdrawn from chemical solution for 3, 7, 8, 9, 10 days, then $15730 \times 730 \mathrm{mcm}$ pictures were made with resolution $1024 \times 1024$ pixels by digital camera. The size was chosen in such a way to avoid effect of overall ball surface curvature on pixel brightness, and the surface pieces can be considered as plane fragments of rough surfaces. Typical photos are shown in Figure 9. Note, that there are no visual features in the photos that could enable to recognize the differences "by eye".

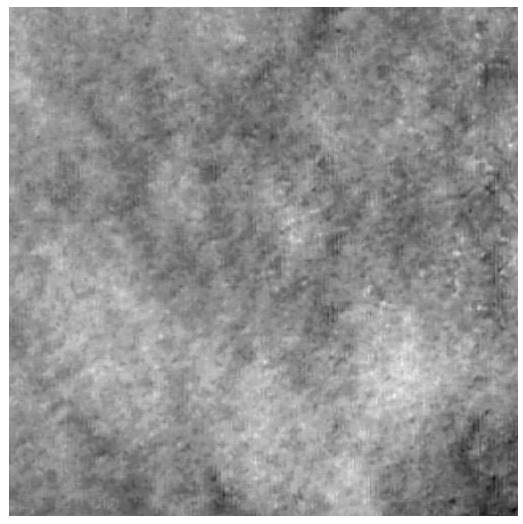

a

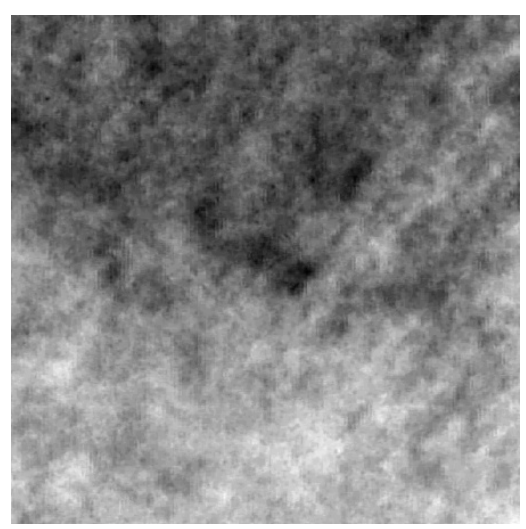

d

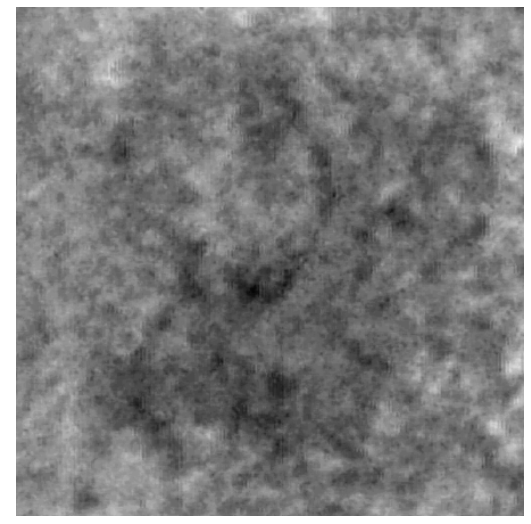

b

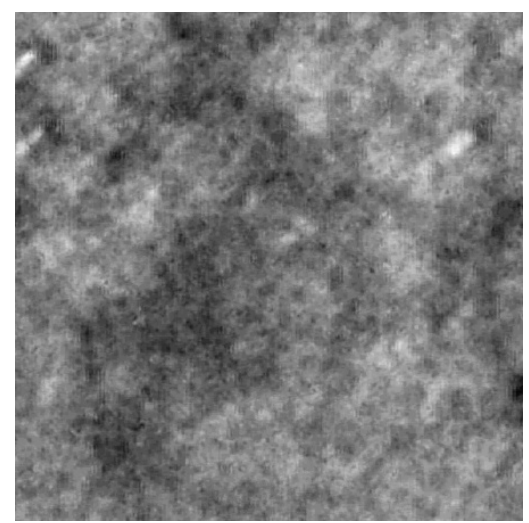

e

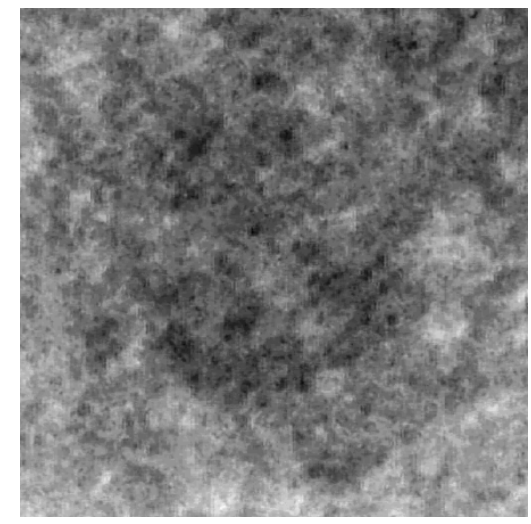

c

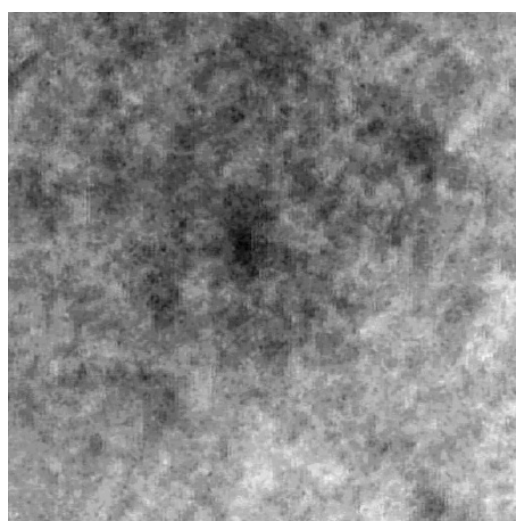

f

Figure 9. Examples of photos of led ball surface pieces $730 \times 730 \mathrm{mcm}$ : a) initial covering before etching (0 days), b-f) coverings after chemical test during $3,7,8,9$ and 10 days, respectively 
The photos obtained were processed by a special program FCP (Formula for Chaos Processing) which enabled to represent photos as surface reliefs - the distributions of effective heights proportional to pixel brightness, set in the points respective to pixel positions in square matrix with discrete step $0.713 \mathrm{mcm}$ in both directions. Typical view of such a relief is shown in Figure 10.

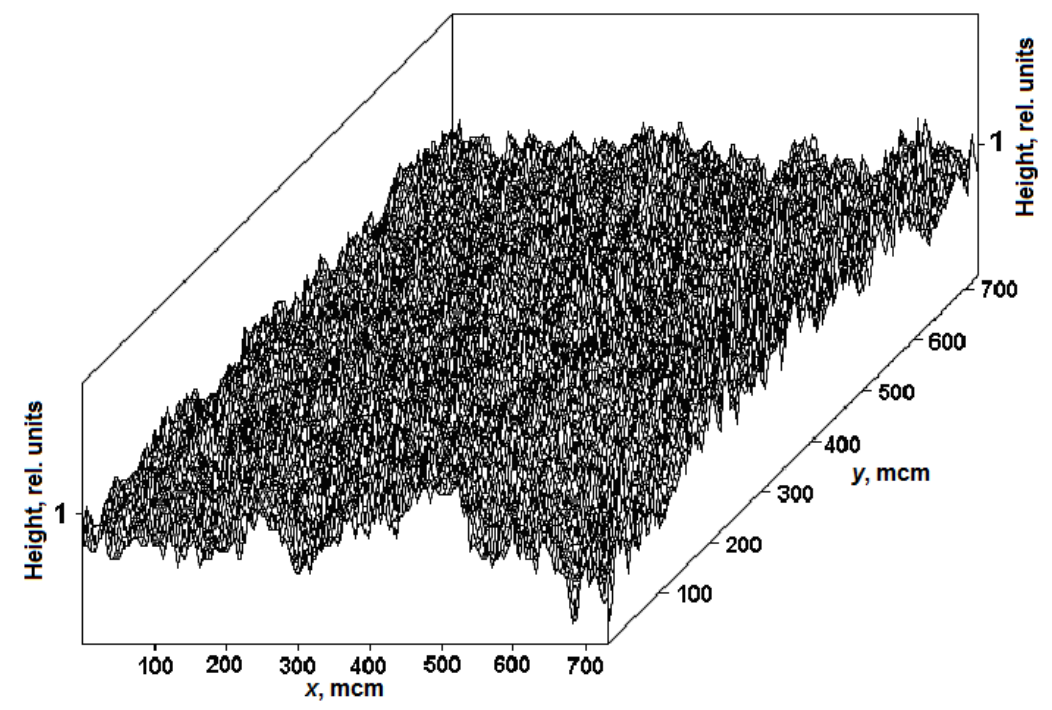

Figure 10. Relief view for the covering before etching, see Figure 9 a (Each $6^{\text {th }}$ point is shown for both the directions $x, y$ )

The heights of such reliefs do not correspond to real roughness, but, as the pixel brightness correspond to local surface slope, reflecting the height gradients, then one can evaluate the scales of covering surface inhomogeneities using such effective reliefs, representing distribution of local reflecting ability of the surface, determined by surface roughness.

Then the reliefs were processed by sm2FNS program realizing SFCAM application to surface reliefs. Two examples of processed relief profiles are shown in Figure 11 for the cases of one and two CLs.

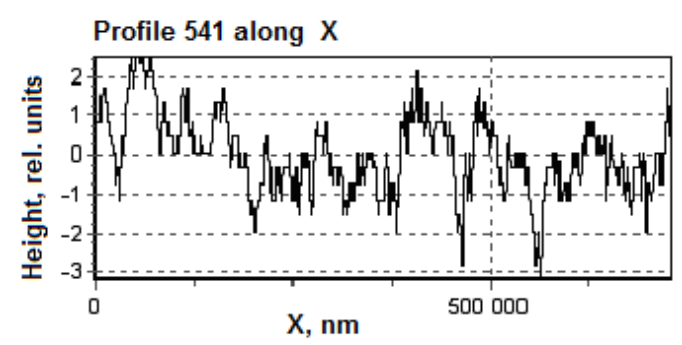

a

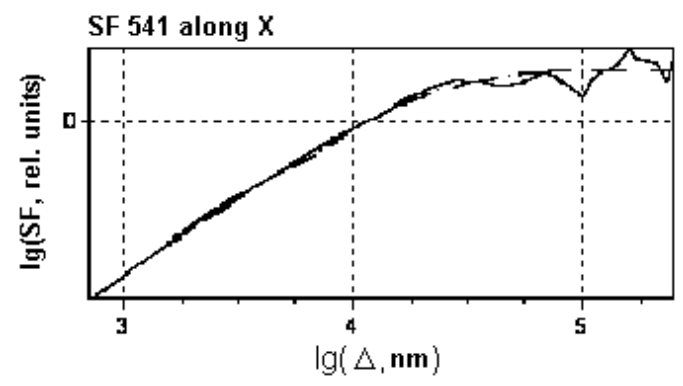

b

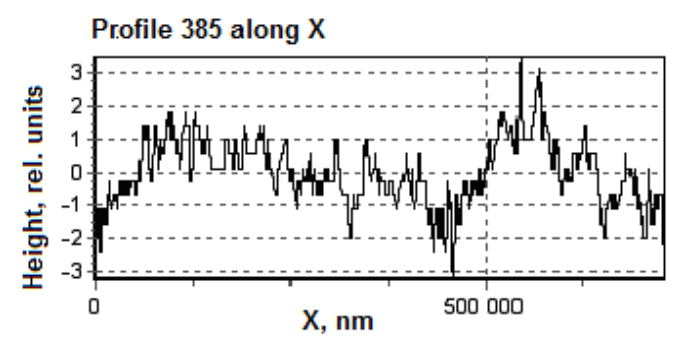

d

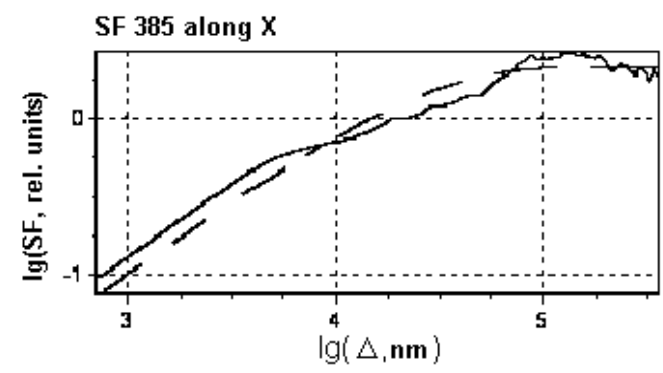

e 


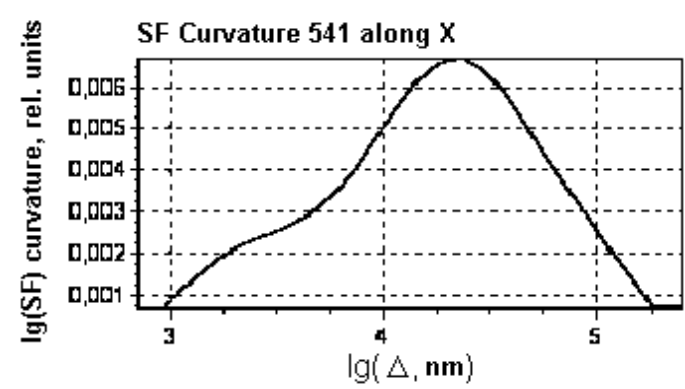

$\mathrm{c}$

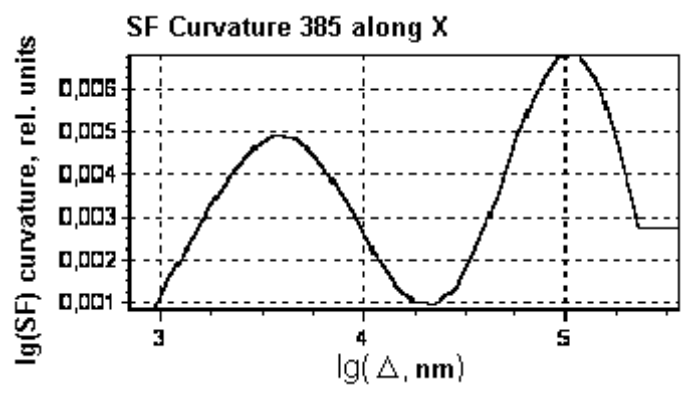

f

Figure 11. Two different profiles of relief in Figure 10 (a, d), their SFs (b, e) and SF curvatures (c, e)

The obtained experimental data had shown that situation was not so simple as in the cases described above, because the individual SFs averaged over relief did not enable to reveal all the observed CLs. Analysis of calculated CLs had shown that the structures of obtained polymer coverings have CLs that can be separated into three classes. Variations of CLs into each class are due to real differences of surface pieces. So, one has to apply statistical methods to determine CLs as the means over registered relief pieces obtained for each etching time during the chemical test. To make the statistical procedure clearer, the results for 3 day etching are presented in Table 1. The maximum relative error of CLs determination can be taken equal to $30 \%$, the CLs from neighboring classes differing by about one order, and so, CLs determination accuracy was sufficient to evaluate CL hierarchy.

Table 1. Summary of CLs obtained for polymer covering after 3 days etching

\begin{tabular}{|c|c|c|c|}
\hline N photo & \multicolumn{3}{|c|}{$L_{1}, \mathrm{mcm} L_{2}, \mathrm{mcm} L_{3}, \mathrm{mcm}$} \\
\hline 1 & 4,32 & 30,1 & 277 \\
\hline 2 & 2,85 & 26,2 & 277 \\
\hline 3 & 2,85 & 26,2 & 277 \\
\hline 4 & 2,48 & 30,1 & 419 \\
\hline 5 & 2,85 & 22,8 & 241 \\
\hline 6 & 2,48 & 26,2 & 241 \\
\hline 7 & 2,85 & 26,2 & 318 \\
\hline 8 & & 22,8 & \\
\hline 9 & & 13,1 & 277 \\
\hline 10 & 4,96 & & 69,2 \\
\hline 11 & & 22,8 & 241 \\
\hline 12 & 1,88 & & 69,2 \\
\hline 13 & 3,28 & 22,8 & 241 \\
\hline 14 & & 19,9 & \\
\hline 15 & 2,48 & 22,8 & 183 \\
\hline CL number & 11 & 13 & 13 \\
\hline Mean CL, mcm & 3,03 & 24,0 & 241 \\
\hline Standard deviation, mcm. & 0,88 & 4,4 & 93,6 \\
\hline
\end{tabular}

The mean CLs for each etching time were calculated in analogous way. The number of revealed CLs varied from 6 (rarely) to 15 , see Table 2. The dependences of CLs on etching time are shown in Figure 12, Table 3. 
Table 2. Numbers of revealed CLs by classes for each etching time

\begin{tabular}{cccc}
\hline Time, days & $L 1$ & $L 2$ & $L 3$ \\
\hline 0 & 0 & 15 & 13 \\
3 & 11 & 13 & 13 \\
7 & 13 & 15 & 6 \\
8 & 10 & 14 & 10 \\
9 & 9 & 14 & 11 \\
10 & 14 & 9 & 6 \\
No covering & 7 & 10 & 13 \\
\hline
\end{tabular}

Table 3. Mean CLs values for each etching time

\begin{tabular}{cccc}
\hline Time, days & $L 1, \mathrm{mcm}$ & $L 2, \mathrm{mcm}$ & $L 3, \mathrm{mcm}$ \\
\hline 0 & & 10,6 & 242 \\
3 & 3,03 & 24,0 & 241 \\
7 & 4,34 & 15,3 & 306 \\
8 & 3,22 & 23,8 & 185 \\
9 & 3,06 & 29,5 & 192 \\
10 & 4,20 & 25,6 & 251 \\
No covering & 1,95 & 34,1 & 204
\end{tabular}
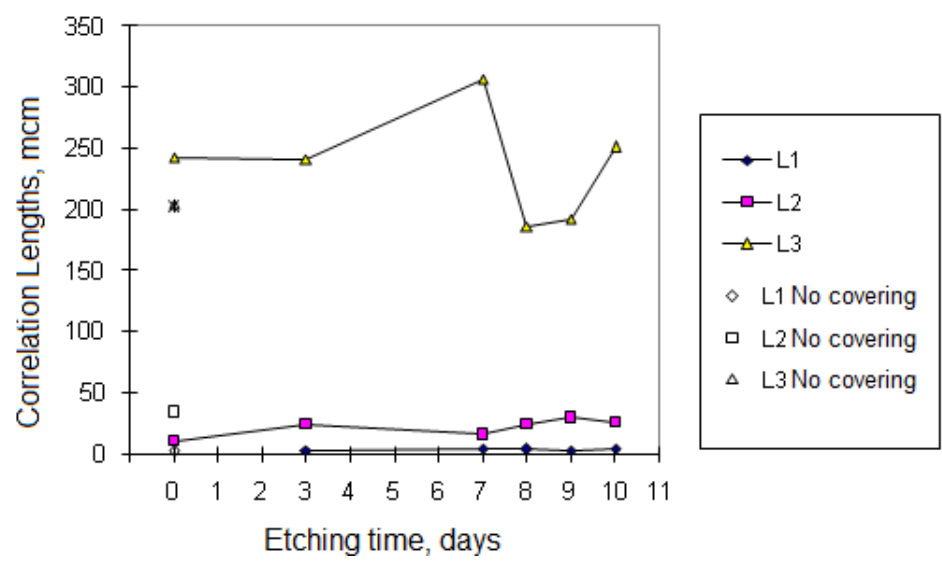

Etching time, days

a
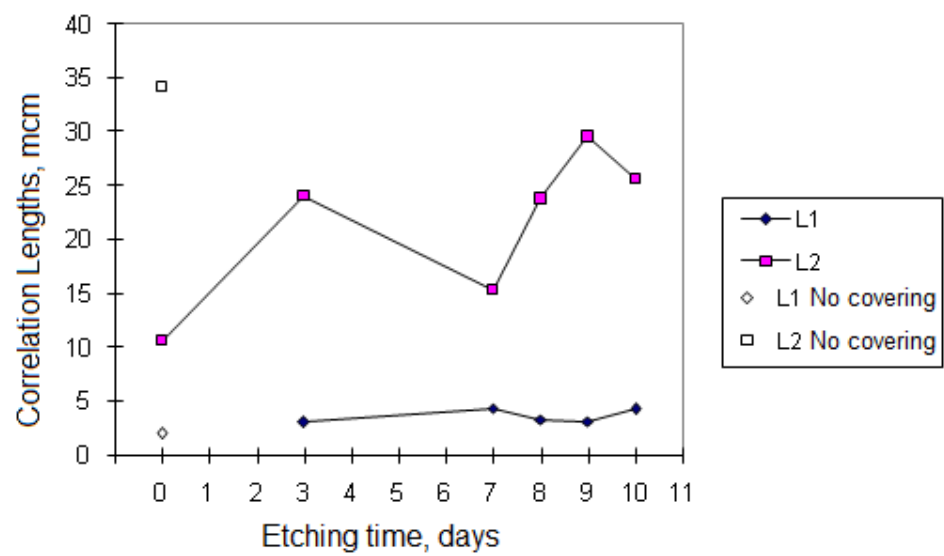

b

Figure 12. Dependence of CLs on etching time, b) being a magnified part of a) for the small CLs 
The calculated results enable one to draw some conclusions about polymer covering surface evolution during the etching process. The greatest CLs $L_{3}=250-300 \mathrm{mcm}$ correspond to the craters, traces of ball collisions taking place during mechanochemical treatment in ball mill. It is seen that the structure of such craters was destructed after 7 days etching when the fast stage of covering destruction was starting. After longer etching the most dense covering fragments were stored on ball surface, that are the fragments corresponding to a skeleton of this structure characterized by CLs $L_{3}=200-250 \mathrm{mcm}$.

CLs $L_{2}=10-35 \mathrm{mcm}$ corresponded to the grained structure of polymer covering due to grain size of initial polymer dust introduced into mill. During chemical etching, the covering destruction seemed to take place on grain boundaries. First, the finest blocks of size $10-15 \mathrm{mcm}$ had removed from the covering that revealed the structure of the greater blocks $(20-30 \mathrm{mcm})$ on covering surface. Destruction of the covering after 7 days etching revealed internal block structure of covering and its skeleton.

CLs $L_{1}=2-5 \mathrm{mcm}$ corresponded to the finest particles of matter independently of its nature (the metal, polymer, or graphite) and the finest craters on covering surface. As is well known, it is impossible to obtain the matter particles smaller than $1 \mathrm{mcm}$ by simple blending using no special means, because the particles smaller than $1 \mathrm{mcm}$ tend to stick together. This problem is solved by using the highly non-equilibrium conditions and special media. In our case the smallest particles were not revealed on the covering before chemical etching. This can be explained in such a way that they were aggregated just after mechanochemical treatment with aggregate size about $10 \mathrm{mcm}$ and greater. During etching these aggregates were broken apart and the smallest particles could be discovered.

Thus, separation of CLs into classes enables to investigate the objects quantitatively on the base of fundamental knowledge about the system and it is most effective when not all the hierarchical levels of system structure can be easily registered by all the data samples. This situation is rather characteristic for natural systems and phenomena.

\section{Conclusion}

Three examples of SFCAM application above are to show that the proposed approach represents a rather subtle tool for investigation of complex systems on the base of various digital data types - time series or surface reliefs treated as the sets of space series. SFCAM, in combination with other well known methods like sliding window method or simplest statistical calculations, enables to register presence of hierarchical organization of the system under study and to evaluate the quantitative characteristics of this organization - the correlation times or correlation lengths. Taking them into account by an order of recognition, from the shortest to the greatest, can assist to reveal the main features of hierarchical structure, that can introduce new physical sense in concrete consideration. And more exact evaluation of hierarchical structures can be made on the base of statistical analysis of correlation characteristics taken into account by separation into classes on the base of general physical knowledge about investigated object. These correlation characteristics enable to evaluate the differences between the real objects due to environmental or experimental conditions, or to follow object's evolution. The proposed "series-by-series" application of SFCAM can be used for analysis of many dimensional objects also without any principal alterations. It should be noted also that SFCAM gave the correct values of half period for model periodic data (Vstovsky, 2006). So, one can obtain evaluations of "persistency length" for periodic or quasiperiodic systems. Note, that SF, being a difference moment, is less sensitive to presence of noise or systematic errors in measured data as compared with other processing methods, that is another advantage of SFCAM.

\section{References}

Bornyakov, S. A., \& Vstovsky, G. V. (2010). First experience of seismodeformation monitoring of Baikal rift zone (by the example of South-Baikal earthquake of 27 August 2008). Doklady RAS, Geophysics, 431(4), $1-5$.

Frisch, U. (1995). Turbulence. The Legacy of A. N. Kolmogorov. Cambridge Univ. Press.

Letnikova, A. F., Vstovsky, G. V., \& Timashev, S. F. (2001). Formation of Anisotropic Fractal Structures during Dissolution of LiF Monocrystals. Materials Science (Medziagotyra) (Kaunas), 7(2), 98-103.

Timashev, S. F. (2011). Phenomenology of Complexity: Information in Chaotic Signals. Russian Journal of General Chemistry, 81(1), 220-233. http://dx.doi.org/10.1134/S1070363211010397

Vstovsky, G. V. (2006). Factual Revelation of Correlation Lengths Hierarchy in Micro- and Nanostructures by Scanning Probe Microscopy Data. Materials Science (Medziagotyra) (Kaunas), 12(3), 262-270.

Vstovsky, G. V. (2008). Revelation of spatial and temporal hierarchical structures in complex systems. In R. M. Yulmetev, A. V. Mokshin, S. A. Demin, \& M. Kh. Salakhov (Eds.). Fluctuations and Noise in Complex 
Systems of Living and Non-living Nature (pp. 441-454). Russia, Tatarstan Republic Ministry of Education and Science, Kazan City: "Shkola" Publishing Center.

Vstovsky, G. V. Solovieva, A. B. Timashev, S. F. Zarkhina, T. S. Timofeeva, V. A., \& Netschadina, L. V. (2006). Revelation of hierarchical structure of mechanochemical polymer coverings of metal balls by optical microscopy data. Zavodskaya Laboratoriya, N12, 24-28. (In Russian)

Vstovsky, G. V., \& Bornyakov, S. A. (2010). First experience of seismodeformation monitoring of Baikal rift zone (by the example of South-Baikal earthquake of 27 August 2008). Nat. Hazards and Earth Syst. Sci., 10, 667-672. http://dx.doi.org/10.5194/nhess-10-667-2010 